\title{
Disciplina de Inspeção Sanitária: Alteração de Cronograma e Avaliação de Graduandos em Medicina Veterinária
}

\section{Sanitary Inspection Discipline: Schedule Change and Evaluation of Veterinary Medicine Students}

\author{
Victor Chiaroni Galvão*, Simone de Carvalho Balian \\ Faculdade de Medicina Veterinária e Zootecnia da Universidade de São Paulo \\ * Autor para correspondência: vchiaroni@gmail.com
}

\begin{abstract}
RESUMO
A disciplina de Inspeção Sanitária dos Produtos de Origem Animal (ISPOA) da Faculdade de Medicina Veterinária e Zootecnia da Universidade de São Paulo passou por várias transformações ao longo do tempo devido aos avanços tecnológicos e científicos. O cronograma da disciplina de ISPOA foi reformulado em blocos de conteúdos estreitamente correlatos ou em blocos em que os assuntos não se relacionavam. Houve a aplicação de uma nova metodologia de avaliação de aprendizagem que ocorreu ao fim de cada bloco de conteúdo através de prova teste múltipla escolha (PTME). A amostra deste estudo foi formada por 168 alunos do $9^{\circ}$ semestre de medicina veterinária entre os anos 2017 e 2018. Foram utilizadas análise estatística descritiva e multivariada (teste $t$ ) dos dados. Os resultados demonstraram que houve aumento das notas entre os anos de 2017 e 2018. O resultado do teste $t$ foi de $t=1,12^{-24}$, ou seja, $p<0,05$ evidenciando que ocorreu diferença estatística entre as médias das notas entre os alunos de 2017 e 2018 . Conclui-se que a organização da disciplina de ISPOA e a aplicação das PTME após os blocos de conteúdo aumentaram o desempenho dos alunos.
\end{abstract}

Palavras-Chave: Inspeção Sanitária; Avaliação; Desempenho; Graduação.

\begin{abstract}
The discipline of Sanitary Inspection of Animal Origin Products (SIAOP) of School of Veterinarian Medicine and Animal Science of University of São Paulo has undergone several transformations over time due to technological and scientific advances. The schedule of the SIAOP discipline was reformulated into blocks of closely related content or in blocks in which the subjects were not related. There was the application of a new learning assessment methodology that occurred at the end of each block of content through multiple choice test (MCT). The sample of this study was formed by 168 students from the 9th semester of veterinary medicine between 2017 and 2018 . Descriptive and multivariate statistical analysis ( $t$ test) of the data were used. The results showed that there was an increase in performance between the years 2017 and 2018 . The result of the $t$ test was $t=1,12^{-24}(p<0.05)$, evidencing that there was a statistical difference between the means of the grades between the students of 2017 and 2018. It is concluded that the organization of the discipline of SIAOP and the application of the MCT after the blocks of content increased the performance of the students.
\end{abstract}

Keywords: Sanitary Inspection; Evaluation; Performance; Undergraduation.

\section{Introdução}

A Faculdade de Medicina Veterinária e Zootecnia da Universidade de São Paulo (FMVZ-USP) possui o princípio fundado sobre as diretrizes de Segurança dos Alimentos da Organização Mundial de Saúde Animal (OIE, 2013): garantir a inocuidade dos alimentos do estábulo à mesa. Portanto, o discente é formado para ter uma visão ampla sobre a cadeia de produção dos alimentos, a fim de intervir com soluções adequadas à Saúde Pública.

A disciplina de inspeção sanitária dos produtos de origem animal (ISPOA) faz parte do conjunto de conhecimentos referentes à Segurança dos Alimentos (LUDÉN, 2007; SMULDERS, 2012). 
A finalidade dessa disciplina presente na maioria dos currículos de escolas veterinárias é promover a Saúde Pública. Em outras palavras, os ensinamentos abordados visam a formas de prevenir que determinado produto de origem animal cause mal ao consumidor.

Os veterinários que atuarão na área ISPOA irão inspecionar desde os animais vivos que foram encaminhados ao abate sanitário até produtos de pescado enlatados. O profissional da ISPOA deve conhecer os programas de autocontrole, tais como Boas Práticas de Fabricação e Análise de Perigos e Pontos Críticos de Controle, além de microbiologia e exame clínico de animais de produção. Além disso, as habilidades humanas se fazem necessárias para motivar e liderar equipes: a comunicação e a capacidade de avaliar comportamentos humanos de risco que implicam a contaminação dos alimentos, como a lavagem de mãos.

\section{Objetivo e Motivação}

Identificar se a reformulação do encadeamento dos conteúdos da disciplina de ISPOA e a aplicação de uma metodologia de avaliação de aprendizagem em alunos de graduação de medicina veterinária foram responsáveis pelo melhor desempenho dos alunos nas notas. A motivação foi estimular os alunos a manterem estudo constante da disciplina ao longo do semestre, além de evitar a pressão de realizar apenas uma prova com grande conteúdo.

\section{Método}

A amostra deste estudo foi formada por 168 alunos do $9^{\circ}$ semestre de medicina veterinária entre os anos 2017 e 2018. Em 2017, foram 80 alunos e, em 2018, 88 alunos.

A disciplina de ISPOA da FMVZ-USP passou por várias transformações ao longo do tempo, devido aos avanços tecnológicos e científicos. Em 2017 a disciplina continha 90 horas de carga horária distribuídas em aulas teóricas, práticas e visitas técnicas. O método de avaliação da disciplina consistia em um trabalho em grupo e uma avaliação final de todo o conteúdo. A disciplina contou com a participação de um aluno de pós-graduação do Programa de Aperfeiçoamento de Ensino da USP (PAE) para o auxílio nas atividades.

Em 2018, a disciplina manteve a mesma estrutura acima apresentada, mas com alterações no encadeamento dos conteúdos e no método de avaliação.

O cronograma da disciplina de ISPOA foi reformulado em blocos: o primeiro formado por conteúdos estreitamente correlatos, e o segundo em blocos em que os assuntos não se relacionavam. Por exemplo, a inspeção ante mortem, post mortem, técnicas de insensibilização e abate de animais de açougue formaram um bloco de conteúdo. Entretanto, a inspeção de produtos de abelha, pescado e produtos não comestíveis formou outro bloco de conteúdo. Com isso, formaram-se quatro blocos de conteúdo ao longo da disciplina.

Uma nova metodologia de avaliação foi adicionada à estrutura da disciplina de ISPOA. A aplicação da metodologia de avaliação de aprendizagem ocorreu ao fim de cada bloco de conteúdo, através de prova teste múltipla escolha (PTME), constituída de quatro a seis questões. $\mathrm{O}$ conjunto de questões pertencia a concursos públicos direcionados a médicos veterinários e questões elaboradas pelo docente responsável pela disciplina. As questões foram selecionadas de acordo com o conteúdo do bloco.

Durante a aplicação da PTME ao fim de cada bloco, os alunos foram dispostos em fileiras intercaladas para realizar a prova sem consulta (I). Ao término da prova o aluno entregou ao PAE o gabarito das questões e permanecia com a folha de questões (II). Os alunos foram instruídos a se agrupar (8-10 pessoas) para discutirem as respostas (III). Terminando a discussão, as questões e as respectivas respostas foram apresentadas em projeção de slides para discussão com o docente e o PAE (IV). O tempo de cada etapa do método foi: (I) 15-20 min., (II) e (III) 5 min., e (IV) 15-30 min. O objetivo da aplicação da avaliação em blocos de conteúdo foi estimular os alunos a testarem os seus conhecimentos sobre o bloco abordado até então. 
As análises estatísticas descritiva e multivariada (teste $t$ ) foram aplicadas nos dados para extrair inferências. Foi utilizada uma planilha eletrônica para a compilação dos dados e a análise estatística.

\section{Resultados e Discussão}

Após a tabulação e a análise dos dados nas planilhas eletrônicas, obtiveram-se os resultados contidos na Tabela 1. É possível notar que houve aumento das notas entre os anos de 2017 e 2018. A diferença entre a menor nota foi de 3,8 pontos, indicando que no ano de 2017 houve nota inferior à média mínima para aprovação na disciplina $(5,0)$. Já entre a maior nota foi de 0,7 pontos. Entre os anos de 2017 e 2018, a média geral da turma aumentou 1,7 pontos, o que a princípio foi significativo.

A Tabela 2 mostra intervalos de nota dos alunos entre os anos de 2017 e 2018 . Vê-se que, em 2017, quatro alunos obtiveram notas inferiores a
5,0 pontos (reprovação). Já em 2018, não houve alunos com nota inferior a 5,0 pontos. No intervalo entre 5,0 e 7,0 pontos, o ano de 2017 apresentou 20 alunos, porém no ano de 2018 não houve alunos nesse intervalo. Já no intervalo entre 7,1 e 9,0, em 2017 foram 55 alunos, e 42 em 2018. Mas quando se observam notas maiores que 9,0 pontos, o ano de 2018 destacou-se, apresentando 46 alunos. Já em 2017, apenas um aluno teve nota superior a 9,0 pontos.

Os dados apresentados até o momento indicam que o desempenho dos alunos aumentou de um ano para o outro. Provavelmente a reformulação do cronograma da disciplina de ISPOA e a aplicação das PTME influenciaram os resultados.

A fim de verificar se houve diferença estatística entre as médias de notas das turmas de 2017 e 2018 , foi aplicado o teste $t$ de Student. A aplicação do teste $t$ adotou intervalo de confiança de $95 \%$, em que a hipótese nula $(\mathrm{H} 0)$ indica que não há

\begin{tabular}{|c|c|c|c|}
\hline Ano & Menor nota & Maior nota & Média da turma \\
\hline 2017 & 3,6 & 9,3 & 7,3 \\
\hline 2018 & 7,4 & 10,0 & 9,0 \\
\hline
\end{tabular}

Tabela 1 - Média alcançada pelos alunos e notas mais alta e mais baixa obtidas entre os anos 2017 e 2018 .

\begin{tabular}{|c|c|c|}
\hline Ano & Intervalo de nota & Número de alunos \\
\hline 2017 & $<5,0$ & 4 \\
\hline & $5,0-7,0$ & 20 \\
\hline & $7,1-9,0$ & 55 \\
\hline Total & $>9,0$ & 1 \\
\hline 2018 & & 80 \\
\hline & $<5,0$ & 0 \\
\hline & $5,0-7,0$ & 0 \\
\hline & $7,1-9,0$ & 42 \\
\hline
\end{tabular}

Tabela 2 - Distribuição das notas dos alunos da turma entre os anos 2017 e 2018. 
diferença estatística entre as médias de notas das turmas de 2017 e 2018, e a hipótese alternativa (H1) significa que existe diferença entre as médias dos alunos (HAIR, 2009).

O resultado do teste $t$ foi de $t=1,12^{-24}$, ou seja, $\mathrm{p}<0,05$. Portanto, rejeita-se $\mathrm{H} 0$ e aceita-se H1. Com isso, pode-se afirmar que houve diferença entre as médias das notas entre os alunos de 2017 e 2018. Este resultado mostra que a organização do cronograma do curso de ISPOA e a aplicação das PTME provavelmente causaram aumento das notas dos alunos.

A intenção de reorganizar a disciplina de ISPOA em blocos de conteúdo foi fruto de avaliações formativas realizadas pelo docente responsável pela disciplina e pelo aluno PAE. A avaliação formativa consiste em buscar aprimorar o processo de ensino-aprendizagem através de erros e acertos discentes e docentes ao longo do tempo, buscando melhorar o desempenho dos alunos (SILVA, 2014; REYNOLDS, 2008; BALLESTER, 2003).

Ao final de cada bloco de conteúdo foi aplicada uma PTME. Sabe-se que testes de múltipla escolha fazem parte da avaliação somativa. A avaliação somativa visa a avaliar se os propósitos, progressos e resultados dos avaliados estão de acordo com os objetivos do programa ou de uma disciplina (REYNOLDS, 2008). Em outras palavras, consiste na soma de um ou mais resultados de avaliação. Silva (2014) afirma que avaliações frequentes e consistentes melhoram o rendimento dos alunos. De fato, o incremento das PTME resultou em aumento do desempenho.

Bollela (2018) apresentou as vantagens e desvantagens do teste múltipla escolha. Entre as vantagens destacam-se a possibilidade de avaliar os alunos em diferentes níveis de complexidade, a facilidade de correção e feedback de desempenho de cada aluno. Essas vantagens favoreceram a metodologia do presente trabalho, daí a escolha dos testes múltipla escolha e não de outra forma de avaliação.

Já as desvantagens seriam a dificuldade em se avaliar a profundidade de raciocínio, a dificuldade de delimitação estreita e precisa em apenas uma questão de tal assunto da disciplina e o acerto casual ou "chute" (BOLLELA, 2018). Realmente não se consegue alcançar os raciocínios em profundidade dos alunos com PTME, mas o intuito das PTME foi aumentar o desempenho dos alunos e a fixação dos conteúdos. A delimitação dos assuntos das questões foi facilitada pela ampla gama de questões de concursos públicos veterinários disponíveis na internet. $\mathrm{O}$ "chute" é uma realidade difícil de coibir, mas que faz parte quando se aplicam avaliações.

\section{Conclusões}

Os resultados obtidos neste trabalho mostram que a organização do cronograma da disciplina de ISPOA e a aplicação das PTME após os blocos de conteúdo, em conjunto com o que já vinha sendo feito ao longo do tempo na disciplina, aumentaram o desempenho dos alunos.

Sugere-se que a organização da disciplina de ISPOA e a aplicação das PTME sejam administradas em outros cursos de medicina veterinária com a finalidade de verificar se esse método é replicável e consistente.

\section{Referências Bibliográficas}

BALLESTER, M. Avaliação como Apoio à Aprendizagem. Porto Alegre: Editora Artmed, 2003.

BOLLELA, V. R.; BORGES, M. de C. \& TRONCON, L. E. de A. "Avaliação Somativa de Habilidades Cognitivas: Experiência Envolvendo Boas Práticas para a Elaboração de Testes de Múltipla Escolha e a Composição de Exames". Revista Brasileira de Educaşão Médica, vol. 4, n. 42, 2018, pp. 74-85.

HAIR, J.; ANDERSON, R. \& BABIN, B. Multivariate Data Analysis: a Global Perspective. $7 \mathrm{ed}$. Upper Saddle River: Prentice Hall, 2009, p. 785.

LUNDÉN, J.; BJORKROTH, J. \& KORKEALA, H. "Meat Inspection Education in Finnish Veterinary Curriculum". Journal of Veterinary Medicine Educational, vol. 2, n. 34, 2007.

REYNOLDS, G. R.; LIVINGSTON, R. B. \& WILLSON, V. Measurement and Assessment in Education. 2 ed. Texas: Editora Pearson, 2008.

SILVA, D. S. G. da; MATOS, P. M. de S. \& ALMEIDA, D. M. de. "Métodos Avaliativos no Processo de Ensino e Aprendizagem: uma Revisão". 
Cadernos de Educaşão | FaE/PPGE/UFPel, n. 47, 2014, pp. 73-84. Disponível em: $<$ http://dx.doi.org/10.15210/ caduc.v0i47.4651>. Acessado em 20 dez. 2018.

SMULDERS, F. J. M.; BUNCIC, S.;
BEHLHABER, K.; HUEY, R. J.; KORKEALA, H.; PRIETO, M. \& STEINHAUSEROVA, I. "Toward Harmonization of the European Food Hygiene/ Veterinary Public Health Curriculum". Journal of
Veterinary Medicine Educational, vol. 2, n. 39, 2012, pp. 169-179.

WORLD ORGANISATION FOR ANIMAL HEALTH (OIE). "Veterinary Education Core Gurriculum". Paris, 2013. Disponível em: $<$ http://www. oie.int/Veterinary_Education_Core_Curriculum.pd $\gg$. Acessado em 27 mar. 2019.

Publicado em 28/07/2020. 